\title{
A monoclonal antibody to ameliorate central nervous system infection and improve survival in a murine model of human Enterovirus-A71 encephalomyelitis
}

\author{
Soon Hao Tan ${ }^{\text {a }}$, Kien Chai Ong ${ }^{b}$, David Perera ${ }^{c}$, Kum Thong Wong a, * \\ a Department of Pathology, Faculty of Medicine, University of Malaya, 50603, Kuala Lumpur, Malaysia \\ b Department of Biomedical Science, Faculty of Medicine, University of Malaya, 50603, Kuala Lumpur, Malaysia \\ ${ }^{\mathrm{c}}$ Institute of Health and Community Medicine, Universiti Malaysia Sarawak, 94300, Kota Samarahan, Sarawak, Malaysia
}

\section{A R T I C L E I N F O}

\section{Article history:}

Received 25 February 2016

Accepted 13 April 2016

Available online 20 June 2016

\section{Keywords:}

Enterovirus A71

Monoclonal antibody

Therapeutic used

Mouse model of human EV-A71

encephalomyelitis

\begin{abstract}
A B S T R A C T
Background: Enterovirus A71 (EV-A71) encephalomyelitis is an often fatal disease for which there is no specific treatment available. Passive immunization with a specific monoclonal antibody to EV-A71 was used on a murine model of EV-A71 encephalomyelitis to evaluate its therapeutic effectiveness before and after established central nervous system (CNS) infection.

Methods: Mice were intraperitoneally-infected with a mouse-adapted EV-A71 strain and treated with a dose of monoclonal antibody (MAb) daily for 3 days on day 1, 2 and 3 post-infection or for 3 days on 3, 4 and 5 post-infection. Treatment effectiveness was evaluated by signs of infection and survival rate. Histopathology and qPCR analyses were performed on mice sacrificed a day after completing treatment Results: In mock-treated mice, CNS infection was established from day 3 post-infection. All mice treated before established CNS infection, survived and recovered completely without CNS infection. All mice treated after established CNS infection survived with mild paralysis, and viral load and antigens/RNA at day 6 post-infection were significantly reduced.

Conclusions: Passive immunization with our MAb could prevent CNS infection in mice if given early before the establishment of CNS infection. It could also ameliorate established CNS infection if optimal and repeated doses were given.
\end{abstract}

(c) 2016 Elsevier B.V. All rights reserved.

\section{Introduction}

Enterovirus A71 (EV-A71), is non-enveloped, single-stranded, sense RNA virus that belongs to the species A group, genus Enterovirus within the Picornaviridae family (Ong and Wong, 2015; Solomon et al., 2010). Outbreaks of EV-A71 associated hand, foot, and mouth disease (HFMD) complicated by acute flaccid paralysis and encephalomyelitis have been reported worldwide (Solomon et al., 2010). These central nervous system (CNS) complications may result in death or severe permanent disability (Huang et al., 1999; Weng et al., 2010). Currently there are no licensed vaccines or antiviral drugs available to prevent or treat EV-A71 infection (Mao et al., 2016; Solomon et al., 2010).

Several studies have previously shown that hyperimmune sera

\footnotetext{
* Corresponding author.

E-mail address: wongkt@ummc.edu.my (K.T. Wong).
}

or monoclonal antibodies (MAb) containing EV-A71-specific neutralizing antibodies were able to protect experimentallyinfected mice from disease ( $\mathrm{Ng}$ et al., 2015; Tan et al., 2014). Recently, we described the effective use of a single optimal dose of hyperimmune serum to prevent and ameliorate CNS infection in a murine model of EV-A71 encephalomyelitis (Ong et al., 2010; Tan et al., 2014). However, the use of hyperimmune sera for passive immunization studies has several disadvantages compared to monoclonal antibodies, including poor reproducibility due to batch-to-batch variability of neutralizing antibody production and specificity.

In this study, we have produced an EV-A71-specific mouse MAb to study its effectiveness to prevent and/or treat EV-A71 infection before or after CNS involvement. Since both skeletal muscles and the CNS were infected in most murine models of EV-A71 infection, the observed limb weakness/paralysis in infected animals could be due either to myositis and/or CNS infection (Ong et al., 2008; Tan et al., 2014). Hence, we believe a more objective way to assess the 